\title{
Design of an electricity consumption measurement system for Non Intrusive Load Monitoring
}

\author{
Sarra Houidi \\ ENIT LSE, Université de Tunis El Manar \\ IREENA, Université de Nantes \\ sarra.houidi@etu.univ-nantes.fr \\ Dominique Fourer \\ IBISC EA 4526, Université d'Évry Val d'Essonne \\ Courcouronnes, France \\ dominique.fourer@univ-evry.fr
}

\author{
François Auger \\ IREENA, Université de Nantes \\ Saint-Nazaire, France \\ francois.auger@univ-nantes.fr \\ Laurence Miègeville \\ IREENA, Université de Nantes \\ Saint-Nazaire, France \\ laurence.miegeville@univ-nantes.fr
}

\author{
Philippe Frétaud \\ IREENA, Université de Nantes \\ Saint-Nazaire, France \\ Philippe.Fretaud@univ-nantes.fr
}

\author{
Houda Ben Attia Sethom \\ ENIT, LSE LRIIES15 \\ Université de Tunis El Manar \\ houda.benattia@enicarthage.rnu.tn
}

\begin{abstract}
Non Intrusive Load Monitoring (NILM) of Home Electrical Appliances (HEAs) has received a major interest in the last twenty years due to the increase of electricity consumption in the residential sector. One NILM key component is the acquistion of voltage and current signals at a high sampling rate, to ease HEAs recognition. In this paper, we discuss the design of a cheap and easy to install current and voltage measurement system based on an Arduino MKR Zero microcontroller. This system allows to monitor the usage of HEAs and thus give to consumers information on their consumption and help them to manage it. The hardware architecture of the proposed system is first described. It includes the current and voltage conditioning circuits. Then, a detail of the software implemented in the microcontroller is given. It consists mainly of an interruption routine that ensures the acquisition regularity and a background task for storing data into a micro SD card. Finally, we show in the last section several individual HEAs power consumption profiles and a whole house power consumption profile obtained using the designed acquisition device.
\end{abstract}

Index Terms-Non Intrusive Load Monitoring (NILM), Home Electrical Appliances (HEAs), data acquisition, Arduino microcontroller, sampling frequency.

\section{INTRODUCTION}

Direct feedback on electricity consumption in the residential sector helps the consumer to get real-time information down to the HEA level [1]. It has proved to induce considerable behaviour changes and substantial energy savings: almost $12 \%$ of reduction in the total residential energy consumption [1], [2]. As an indicator, if broadly implemented in United States, real-time feedback could provide the equivalent of 100 billion kilowatt-hours of electricity savings annually by 2030 [1]. Supervised Non Intrusive Load Monitoring (NILM) has recently emerged as a promising solution to provide a breakdown of the residential energy consumption without instrumenting all Home Electrical Appliances (HEAs) [3], [4]. It involves several steps detailed in Fig. 1] [3], [5], [6]. The first step consists of acquiring voltage and current measurements at the Point of Common Coupling (PCC). In the second step, electrical features such as active and reactive powers and/or their harmonics are extracted from voltage and current measurements. The next step involves the detection of events in the aggregated data due to one HEA switching On or Off (or changing state). The fourth step is the identification of HEAs in the load curve through pattern recognition based machine learning techniques which require prior knowledge about HEAs and imply the establishment beforehand of a HEAs database [6]. This database consists of HEAs that are described with electrical features derived from the voltage and current measurements acquired with the acquisition system. Finally, an estimation of each HEA contribution in the load curve is achieved. These informations should be directly fed back to the consumers, so that they can make an informed choice about saving energy and hence reducing their electricity bills. This paper focuses on the upstream of NILM methods: the design and development of a low cost and and easy to install metering unit for real-time current and voltage acquisition. The paper is organized as follows: Section II presents a state of the art of the acquisition systems commercially available, as well as the ones designed for academical and research purposes. This state of the art allows to set up a number of requirements for the design of the developed monitoring device and to stress the characteristics of the system that are innovative in comparison with what is available. Section III introduces the proposed acquisition system by first giving a general overview of its architecture before describing in detail the hardware architecture including the current and voltage conditionning stages, and the software which mainly includes and interrupt service routine implemented in an Arduino MKR Zero microcontroller for real-time acquisition and data storage. The sampling rate selection will be detailed in addition to a data compression technique that allows to store more measurements in the microcontroller's RAM and then in the micro SD card. Section IV shows real acquistions obtained using the proposed prototype: individual HEAs and a whole house power consumption profiles. Finally, Section V is devoted to the final conclusions and pointers to research directions and improvements. 


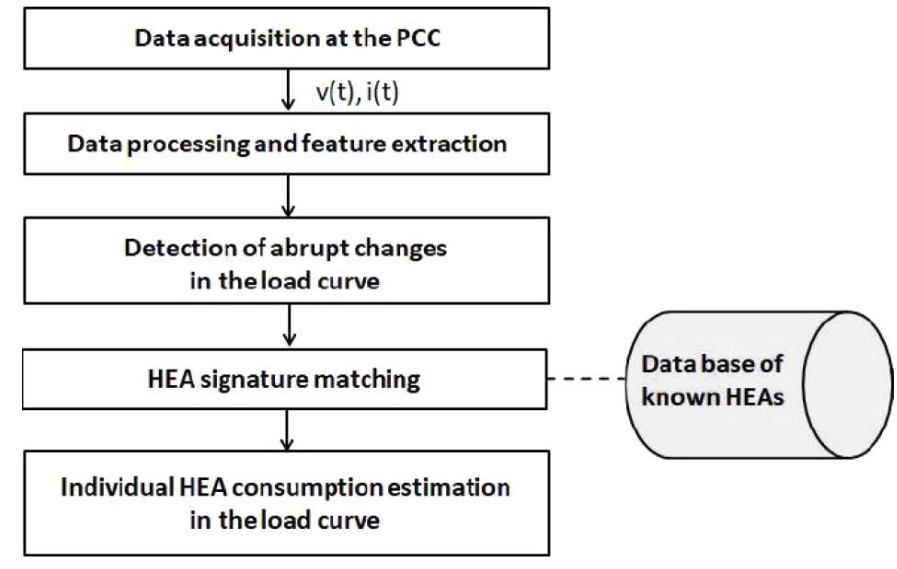

Fig. 1. General framework of supervised NILM methods

\section{STATE OF THE ART}

The role of a data acquisition system is to obtain current and voltage measurements at an appropriate rate so that distinctive HEAs' signatures can be identified in the load curve [7]. Line voltages and currents are sensed and supplied to a microcontroller to allow data logging and storage. One of the main concern for the developpement of such a device is the sampling rate that somehow "governs" its cost. Indeed, it determines the type of information that can be extracted from the electrical signals as well as the ability of the device to support long-time measurements [8]. For instance, to capture the higher order harmonics of the electrical signals, which are integer multiples of the fundamental frequency, the sampling rate of the meter must satisfy the Shannon sampling theorem. To capture transient events or electrical noises generated by the electrical signals, frequencies from the kilohertz range are needed [3], [7]. Conversely, a low sampling frequency allows to store an important duration of measurements. Recently, the world of the industry as well as researchers have taken a close interest in manufacturing these devices, taking into account these considerations. In what follows, we present some of the industrial marketed products before presenting some of the prototypes set up by researchers for experimental evaluation. We aim at highlighting the main requirements for our own prototype design.

\section{A. Industrial marketed products}

While not flooding the market, energy monitoring products are now more and more widespread. They aim at providing the needed feedback to consumers by monitoring the whole-house energy consumption. We are interested into products that are as close as possible to what we aim at designing. Thus, products such as "The Energy Detective 5000 (TED 5000)" [9], "Wattseeker by Qualisteo" [10], [11], "Eyedro" [12], "Neurio" [13] and "Sense Home Energy Monitor" [14] are concerned. They combine a hardware device (hereinafter referred as a "Measurement Transmission Unit (MTU)") and a web-based analytical tool. The hardware device is installed in the electric panel with Current Transducers (CTs) clamped around the phases of the power supply cable in addition to a voltage transducer. For some products, professionals are needed for the installation. The voltage and current measurements are transmitted to a local PC or a remote server (generally a cloudbased server) through an Ethernet gateway, Wi-Fi or Power Line Communication (PLC) technology. Table I presents the main characteristics of these products. It is to be noted that the approximated prices don't take the costs of the installation into account when required.

\section{B. Research prototypes}

Few non-commercial metering devices were developped as prototypes by the NILM research community. For most of the researchers, the design of these prototypes is motivated by limitations, especially in terms of sampling rate and cost, found in available commercial solutions. For instance, K. Gomez et al. introduce in [15] "Energino" a novel hardware and software solution for realtime energy consumption monitoring in wireless networks. It consists of an hardware and an Arduino platform-based software. A management backend written in Python is used to gather the energy consumption statistics. The hardware stage integrates a voltage sensor (based on a voltage divider) and a current sensor (based on the Hall effect). The Arduino continuously polls the voltage and the current sensors and accumulates the values into two separate registers. At the end of the polling period the average power consumption is computed and the result is sent through an USB to an interface. Another solution for the real-time energy management in a house is presented in [16]. It consists of several slave STM32 microcontrollers with their voltage and current transducers, connected to HEAs and which communicate via PLC with a master data aggregator. The slave microcontrollers are programmed to acquire the voltage and current with two simultaneous 12 bits resolution analog to digital converters at a $10 \mathrm{kHz}$ sampling frequency. Through an implemented web server, the users can remotely control their consumption using a web browser. M.C. Lorek et al. present in [17] a low-cost prototype of small size peel-and-stick electricity meter PCB comprising current and voltage sensors with their transducer circuits and a Texas Instruments MSP430G2131 microcontroller. The prototype is installed on the external face of the electrical panel to generate voltage and current signals at a sampling rate of $1920 \mathrm{~Hz}$. It does not require any intervention of a professional.

Based on this literature, we have been able to set the criteria that make our prototype an efficient alternative. The criteria consist of:

- a sampling rate that allows to extract harmonics up to the $15^{\text {th }}$ range (higher than $1.5 \mathrm{kHz}$ according to Shannon theorem) and at the same time, that supports long-time measurements,

- a good resolution,

- a low cost and a low power device, cheap to produce and to deploy, 
TABLE I

COMMERCIALLY AVAILABLE ENERGY MONITORING DEVICES.

\begin{tabular}{|c|c|c|c|c|c|}
\hline & TED 5000 & Eyedro & Neurio & Sense Home Energy & WattSeeker \\
\hline Installation & $\begin{array}{l}\text { MTU + CTs in } \\
\text { the electric panel } \\
\text { (by a professional) }\end{array}$ & $\begin{array}{l}\text { MTU + CTs in } \\
\text { the electric panel } \\
\text { (by users) }\end{array}$ & $\begin{array}{l}\text { MTU + CTs in } \\
\text { the electric panel } \\
\text { (by users) }\end{array}$ & $\begin{array}{c}\text { MTU + CTs in } \\
\text { the electric panel } \\
\text { (by a professional) }\end{array}$ & $\begin{array}{l}\text { Lynx Datalogger } \\
\text { (by a professional) }\end{array}$ \\
\hline $\begin{array}{c}\text { Software } \\
\text { support }\end{array}$ & $\begin{array}{c}\text { Google Power meter / } \\
\text { Bidgely /Plottwatt }\end{array}$ & My Eyedro software & $\begin{array}{l}\text { Neurio mobile app } \\
\text { and Web service }\end{array}$ & Sense mobile app & Wattseeker software \\
\hline $\begin{array}{c}\text { Network } \\
\text { communication }\end{array}$ & $\begin{array}{c}\text { Ethernet (IP protocol) } \\
+ \text { wireless }\end{array}$ & $\begin{array}{c}\text { Ethernet (IP protocol) } \\
+ \text { wireless }\end{array}$ & WLAN access & WLAN access & Ethernet (IP protocol) \\
\hline $\begin{array}{l}\text { Sampling } \\
\text { frequency }\end{array}$ & $1 \mathrm{~Hz}$ & N/A & $4 \mathrm{kHz}$ & $1 \mathrm{~Hz}$ & $2 \mathrm{kHz}$ \\
\hline Approximate price & $\$ 200$ & $300 \$ C A$ & $300 \$ C A$ & $\$ 300$ & N/A \\
\hline Origine & USA & Canada & Canada & USA & France \\
\hline Company & The Energy Detective (TED) & Eyedro Green Solutions & Neurio & Sense & Qualisteo \\
\hline
\end{tabular}

- an autonomous device that can operate as a standalone monitor, with a dedicated power supply,

- an easy installation.

In what follows, the main components of the proposed current and voltage acquisition prototype are presented.

\section{ARCHITECTURE OF THE PROPOSED ACQUISITION SYSTEM}

This section is devoted to the description of the hardware and software part of the proposed current and voltage measurement device. We first give a general overview of our prototype architecture.

\section{A. Acquisition system overview}

The acquisition system that we set up, includes the key components of an acquisition and data processing chain. The general architecture of the system can be seen in Figure 2 a) and a photo of the real acquisition device is shown in Figure 2(b). The whole system is spread over three boards, so the sensors or the microcontroller can easily be changed. In what follows, the hardware subsystem and the software implemented in the Arduino MKR Zero microcontoller are described in detail. The current and voltage measurement board includes a Chauvin Arnoux E3N probe for current sensing and a LEM LV25-P for voltage sensing, whose output quantities are adapted to the voltage range (0-3.3V) expected by the Analog to Digital Conversion (ADC) included in the Arduino MKR Zero microcontroller through conditioning circuits. This board allows the measurement of a consumption which is almost equal to $6 \mathrm{~kW}$. This corresponds to the maximum power allowed by a usual dwelling contract from the French electricity provider. The microcontroller is devoted to the ADC and to data sampling and storage in a micro SD card. The entire system is plugged into a nearby power outlet that delivers $230 \mathrm{~V}$ AC. This is connected to a power supply circuit that incorporates a $2 \times 12 \mathrm{~V}-5 \mathrm{VA}$ transformer whose output is given to a bridge rectifier that converts the $\mathrm{AC}$ to $\mathrm{DC}$, and delivers the voltage to a filter circuit that smoothes out the ripples and passes it to two voltage regulators IC 7812 and IC 7912, which regulate the output voltage at $+12 \mathrm{~V}$ and $-12 \mathrm{~V}$ DC, respectively. This supply board allows

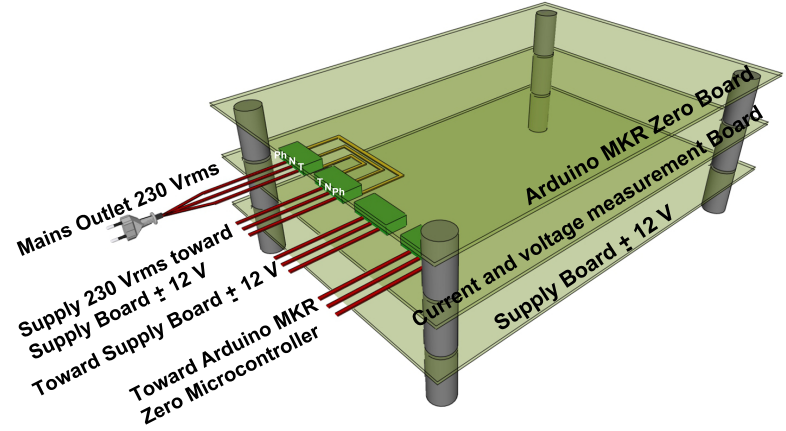

(a)

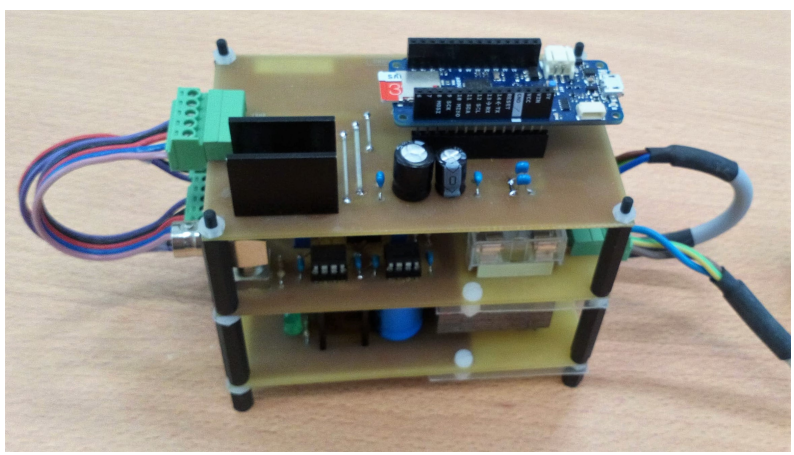

(b)

Fig. 2. Acquisition system overview: General architecture sketch (a) Picture of the real device (b).

to hold the output voltage at $\pm 12 \mathrm{~V}$ necessary for the LEM LV5-P voltage sensor and the Arduino microcontroller that is supplied with $5 \mathrm{~V}$ using an IC 7805 voltage regulator. Protection against overcurrent and short circuits is provided by fuses. This solution remains low cost as shown in Table II which gives a simplified bill of the main components of the proposed prototype when considering wholesale prices.

\section{B. Hardware: current and voltage sensing and conditioning stage}

In order to shape the signals measured by the current and voltage sensors for the ADC, both signals are conditioned. The outputs of both sensors have to be scaled for $0-3.3 \mathrm{~V}$ which represents the voltage range expected by the Arduino 
TABLE II

SIMPLIFIED BILL OF THE MAIN COMPONENTS OF THE PROPOSED ACQUISITION SYSTEM.

\begin{tabular}{|l|c|}
\hline Component & Wholesale price \\
\hline Hall effect voltage transducer LEM LV25-P & $35 €$ \\
\hline Arduino MKR Zero & $21 €$ \\
\hline $4 \times$ operational amplifiers OP27 & $6 €$ \\
\hline $3 \times$ IC voltage regulators 7812,7912 and 7805 & $5 €$ \\
\hline EVO Plus Samsung microSD card $(32 \mathrm{~GB})$ & $15 €$ \\
\hline Total & $\mathbf{8 2} €$ \\
\hline
\end{tabular}

MKR Zero ADC. This is done through electrical conditioning circuits which are detailed in what follows.

1) Current conditioning: Fig. 3 shows the current conditioning circuit. A Chauvin Arnoux E3N current probe is used to measure the current on one of the domestic network phases. This allows to measure the load current without opening the network to insert an ammeter. A voltage $V_{i I}=s i$ is delivered by the probe, where $i$ denotes the load current and $s$ is the probe sensitivity. This voltage constitutes the input of a non-inverting opamp circuit (using an OP27 op. amp.) including the resistors $R_{I 1}$ and $R_{I 4}$. The output voltage $V_{B}=\left(1+\frac{R_{I 1}}{R_{I 4}}\right) V_{i I}$ is applied to a voltage divider made of resistors $R_{I 2}$ and $R_{I 3}$. When the current $i_{+}$is negligible, then:

$$
\frac{V_{B}-V_{o I}}{R_{I 3}}+\frac{V_{\text {ref }}-V_{o I}}{R_{I 2}}=0 \quad A
$$

$R_{I 2}$ and $R_{I 3}$ values are equal so that $V_{o I}=V_{\text {ref }} / 2$ when $i=0 \mathrm{~A}$. A voltage-follower-type opamp circuit is then used to constitute an impedance adaptation stage. The output voltage $V o_{I}$ is hence equal to:

$$
V_{o I}=\frac{R_{I 3}}{R_{I 2}+R_{I 3}} V_{\text {ref }}+\frac{R_{I 2}}{R_{I 2}+R_{I 3}}\left(1+\frac{R_{I 1}}{R_{I 4}}\right) s i
$$

In order to get an output voltage scaled for $0-3.3 \mathrm{~V}$ and a maximal active power close to $6 \mathrm{~kW}$, the probe sensitivity is set at $s=10 \mathrm{mV} / \mathrm{A}$ and the non-inverting opamp circuit as well as the voltage divider have been designed using standard resistor values such as $R_{I 2}=R_{I 3}=R_{I 4}=1 \mathrm{k} \Omega$ and $R_{I 1}=8.25 \mathrm{k} \Omega$. Such values allow to measure a maximal peak value of the current equal to $i_{\max } \approx 35.67 \mathrm{~A}$ which corresponds to an effective value of $i_{\mathrm{rms}} \approx 25.23 \mathrm{~A}$ (when $V_{o I}=3.3 \mathrm{~V}$ ), and thus a maximal value of the active power equal to $P_{\max }=230 i_{\max } / \sqrt{2} \approx 5802 \mathrm{~W}$. Besides, since the Arduino board supports input channels using a 10bit ADC, it maps input voltages between $0 \mathrm{~V}$ and $3.3 \mathrm{~V}$ to integer values between 0 and 1023. Thus, the resolution is $q_{V}=\frac{3.3}{2^{10}} \mathrm{~V}$ per unit. This allows to get a current granularity of $q_{I}=\frac{q_{V}}{s} \frac{1+R_{I 3} / R_{I 2}}{1+R_{I 1} / R_{I 4}} \approx 69 \mathrm{~mA}$ which leads to an active power granularity of $q_{P}=\frac{q_{I}}{\sqrt{2}} 230 \approx 11.3 \mathrm{~W}$, enough to catch dynamics such as low-energy lamp switch on.

2) Voltage conditioning: The voltage measurement is realized thanks to a LEM LV 25-P voltage sensor, which is a closed loop current transformer using Hall effect sensor with galvanic isolation. It allows a voltage measurement up to 500 V. Fig. 4 shows the voltage conditioning circuit. According to

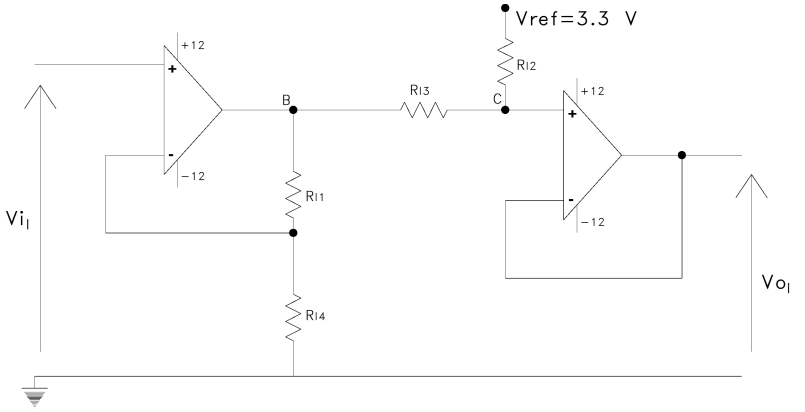

Fig. 3. Current conditioning circuit

the datasheet of this voltage sensor [18], the maximum primary current $i_{\mathrm{pmax}}$ is chosen equal to $10 \mathrm{~mA}$. For this maximum to be obtained when the input voltage is equal to $V_{\mathrm{iNmax}}=$ $230 \sqrt{2} \mathrm{~V}$, we chose $R_{U 1}=\frac{V_{i N \max }}{i_{\text {max }}}=\frac{230 \sqrt{2}}{1010^{-3}} \approx 32.5 \mathrm{k} \Omega$. This value can be approximated using 3 resistors of $100 \mathrm{k} \Omega$ in parallel. Since the conversion ratio is of 2.5 [18], the maximum secondary current equals $i_{\text {smax }}=2.5 \frac{V_{i N \max }}{R_{U 1}}=24.4 \mathrm{~mA}$ can be translated to voltage by the measurement resistor $R_{M}$ between the output and ground. The value of the resistor $R_{M}$ should range between $30 \Omega$ and $190 \Omega$ [18]. In order to scale the measured voltage so that $[-230 \sqrt{2} ; 230 \sqrt{2}] \mathrm{V}$ translates to $[0 ; 3.3] \mathrm{V}$, two opamp voltage follower circuits and a resistive voltage divider are used to obtain $V_{o N} . R_{U 2}$ and $R_{U 3}$ are both fixed to $1 \mathrm{k} \Omega$ so that $V_{o N}=\frac{V_{\text {ref }}}{2}$ when $V_{i N}=0 \mathrm{~V}$. The output voltage $V_{O N}$ is then given by

$$
\begin{aligned}
V_{o N} & =\frac{R_{U 3}}{R_{U 2}+R_{U 3}} V_{\text {ref }}+\frac{R_{U 2}}{R_{U 2}+R_{U 3}}\left(2.5 \times \frac{R_{M}}{R_{U 1}} V_{i N}\right) \\
& =\frac{V_{\text {ref }}}{2}+1.25 \frac{R_{M}}{R_{U 1}} V_{i N}
\end{aligned}
$$

Choosing $R_{M}=130 \Omega$ leads to a maximum value of the output voltage equal to $V_{\mathrm{oNmax}}=3.17 \mathrm{~V}$, which is lower than $3.3 \mathrm{~V}$, the maximum voltage allowed by the ADC. One of the most interesting features of the proposed prototype is the implementation of the resistors on integrated circuit supports, which allows to easily change the power level when needed.

C. Software: analog to digital conversion, sampling and data storage

The analog current and voltage signals provided by the measurement board are digitized by an Arduino MKR Zero microcontroller (32 bit processor SAMD21 Cortex-M0 microcontroller, $48 \mathrm{MHz}$ ), programmed using the $\mathrm{C} / \mathrm{C}++$ programming language, and including ADC channels with 8/10/12 bit resolution. The Arduino MKR Zero microcontroller was chosen for its low cost and performances [19]. Moreover, it integrates a micro-SD card shield and a real-time clock module. As mentionned earlier, one of the main issues for data acquisition is the sampling rate. In the proposed prototype, the current and voltage measurements are sampled at $F_{s}=6.25$ kHz. For this, two libraries are used: "FastADC.h"(compatible with a 10-bit resolution) for ADC management and "SAMDTIMER. $h$ " for timer-triggered interrupts. 


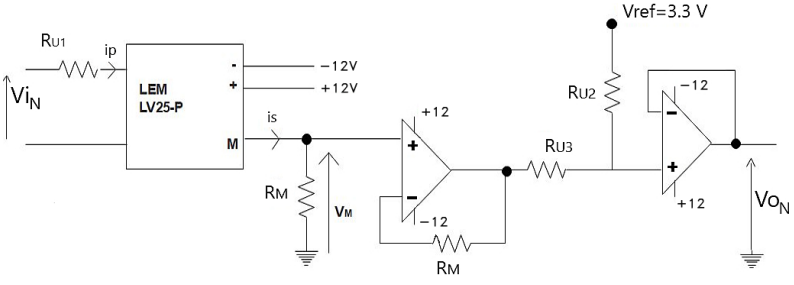

Fig. 4. Voltage conditioning circuit

This sampling frequency is related to the writing time of the SD card. Indeed, in earlier versions of the code, we planned to directly write data into text files in the SD card. However, an analysis of the data writing time in a text file of the SD card showed that the use of the file.print() function of the SD.h library is very time-consuming and takes more time when the amount of data increases. In order to address this issue, instead of using a text file writing system, the SD card is used as an EEPROM composed of 512-byte lines (called "blocks"). The voltage and current acquisitions are performed each time an interrupt service is triggered by a 16-bit timer. We use 20 buffers of 510 bytes to store the current and voltage samples in the interrupt routine. Five bytes are used to store two voltage acquisitions and two current acquisitions. A buffer of 510 bytes contains hence $510 / 5 \times 2=204$ current and voltage acquired samples. When a buffer is full, the next buffer is filled. At the same time, it is signaled to the main loop of the program that the full buffer must be saved in the SD card. This is done via an array of 20 Boolean variables. A boolean variable is set to 1 when a buffer is fully filled, and is set to 0 when this buffer has been stored in a block of the SD card. The use of 20 buffers in the interrupt routine allows to have more time for a buffer of 510 bytes to be written in a line of the SD card using the function "cardwriteBlocks()" of the library "Sd2Fat.h". Indeed, the time required to fill a buffer is $204 / 6250 \approx 32.6 \mathrm{~ms}$, and the average time for writing in a line of the SD card is $4 \mathrm{~ms}$ for the chosen SD card. However this time is not always constant and exceeds sometimes the $40.8 \mathrm{~ms}$ needed to fill a buffer. A comparison of several SD card performances in terms of required time for successively writting 510 bytes into the SD card led to the choice of an SD card with the least amount of time [20]. We have chosen a 32 Go UHSI speed class Samsung EVO Plus SD card that has a reading speed of $95 \mathrm{MB} / \mathrm{s}$, a writing speed of $20 \mathrm{MB} / \mathrm{s}$ and a lifetime of 10000 writing cycles. With the chosen sampling rate, data acquisition can be made during $\frac{204 \times 32.2^{30}}{6250 \times 512}$ seconds which corresponds to 25.4 days. It should be noted that the first line of the SD card is used as a header to store the total number of acquisitions, the date and time and the number of the last completed block. The main loop also manages a user interface. For this, two push buttons are used. One allows to start/stop an acquisition and the other allows to start/stop the transmission of the recorded data on the SD card to a PC via the USB connection cable. The user has access to the information of the SD card (date and time, first and last "block" of an acquisition) and can select the line or lines he wants to transmit.

\section{EXPERIMENTAL RESULTS}

In this section, some acquisitions obtained thanks to the proposed prototype are presented. We show that with the same equipment, it is possible to obtain the consumption profiles of individual HEAs as well as the one of a complete dwelling. These measurements allow to build a database for HEAs recognition and power consumption profile establishment. Here, a power consumption profile corresponds to the time varying active power signal. It is calculated from the current and voltage measurements as follows:

$$
P[n]=\frac{1}{M} \sum_{k=n-M+1}^{n} v[k] i[k]
$$

where $F_{0}=50 \mathrm{~Hz}$ is the utility frequency and $M=F_{s} / F_{0}=$ $\frac{6250}{50}=125$ is the number of samples per period. Fig. 5 shows the consumption profiles of two HEAs.
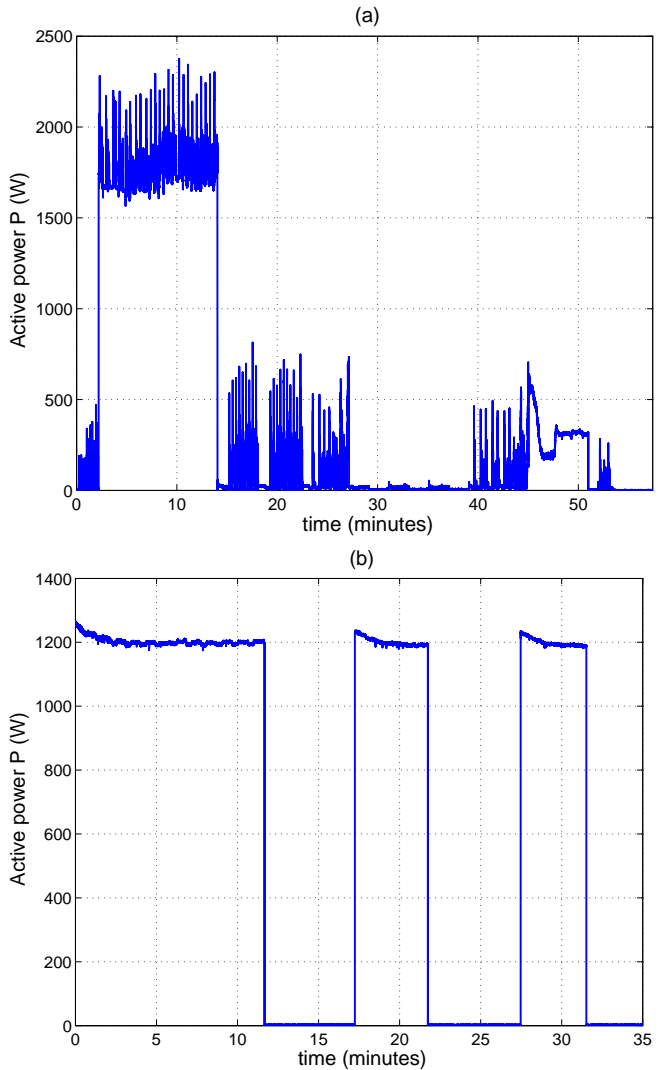

Fig. 5. Individual HEAs power consumption profiles: Washing machine (a) Electric oven (b).

Fig. 5 (a) shows the consumption profile of an LG washing machine operating at $30^{\circ} \mathrm{C}$ in "Rapid" mode with a spin of 800 cycles per minute. In this profile we recognize the different steps of a washing machine operating cycles: water heating during the first fifteen minutes, then rinse from 15 to almost 30 minutes and finally spinning during the last minutes. The 
consumption profile of an electric oven operating at $250{ }^{\circ} \mathrm{C}$ is also presented in Fig. 5 (b), where the on/off control can be observed. On the other hand, Fig. 6 hightlights a twelve hours acquisistion of a whole house power consumption. Out of concern for visibility, only four hours of the whole day power consumption profile are shown. During two hours and a half, the washing machine is operating. At almost one hour and half a LCD TV is activated, followed by the electrical oven that is activated twice at almost the second hour and then the fourth one. Finally, an electrical heater is activated between the second hour and half and the third one. The constant power consumption of almost $250 \mathrm{~W}$, referred as the baseload, corresponds to the two continuously operating wifi boxes, and the standby power consumption of five cell phone chargers plugged in and two TV receivers.

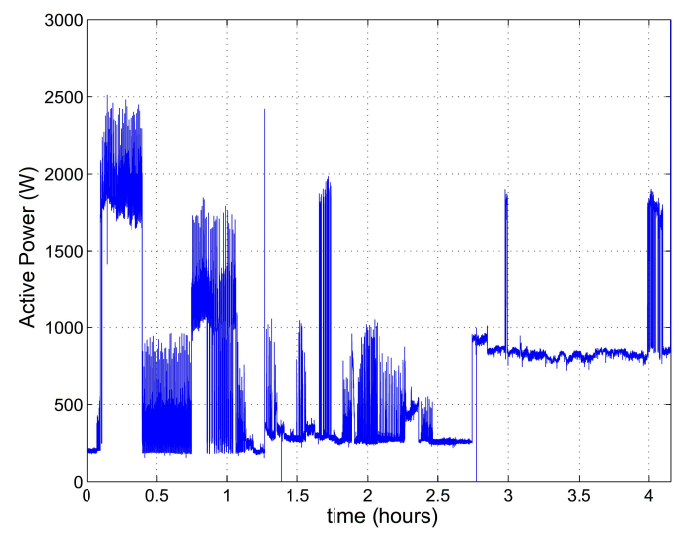

Fig. 6. 4 hours long power consumption profile of a real house.

\section{CONCLUSION}

In this paper, we present a low cost and easy to install device for current and voltage acquisitions in the NILM framework, based on an Arduino MKR Zero microcontroller. The goal of such a device is to acquire voltage and current measurements that will be used to detect the switching on/off of HEAs [21] and to identify them once their electrical signatures have been set up [22]. Energy monitoring devices available in the market as well as prototypes set up by the NILM research community were presented with special emphasis on their main technical characteristics. This state of the art made us able to set the criteria needed for an efficient low cost acquisition system with a higher sampling rate than what is available. We then present the general architecture of the proposed protype before detailing the current and voltage sensing and conditioning hardware boards. We also describe the software and argue the sampling rate choice which is mainly dependent on the data storage speed in the micro SD card. This software can be found online at [23]. In the last section, some of the acquisitions made on individual HEAs and in a whole house are presented. This shows the main originality of this prototype which is its ability to adapt to different power levels. In future, two aspects could be developped: networking functionalities (e.g. Ethernet, WLAN, ZigBee) so that the proposed prototype can provide consumers with information on their consumption through an interface via a web server, and the ability to measure the currents and voltages of a domestic three-phase network.

\section{ACKNOWLEDGMENT}

The authors would like to thank the Tunisian Ministry of High Education and Scientific Research, under Grant LSEENIT-LR11ES15 of University of Tunis El Manar.

\section{REFERENCES}

[1] V. Tiefenbeck, A. Wörner, S. Schöb, E. Fleisch, and T. Staake, "Realtime feedback promotes energy conservation in the absence of volunteer selection bias and monetary incentives," Nature Energy, Nov. 2018.

[2] J. Kelly and W. Knottenbelt, "Does disaggregated electricity feedback reduce domestic electricity consumption? a systematic review of the literature," in 3rd Int. NILM Workshop, May 2016, pp. 14-15.

[3] M. Zeifman and K. Roth, "Non intrusive appliance load monitoring: Review and outlook," IEEE Transactions on Consumer Electronics, vol. 57, no. 1, pp. 76-84, Feb. 2011.

[4] I. Abubakar, S. N. Khalid, M. W. Mustafa, H. Shareef, and M. Mustaph, "Recent approaches and applications of non-intrusive load monitoring," ARPN Journal of Engineering and Applied Sciences, vol. 11, pp. 46094618, Apr. 2016.

[5] G. Hart, "Nonintrusive appliance load monitoring," in Proc. of the IEEE, vol. 80, Dec. 1992, pp. 1870-1891.

[6] S. M. Tabatabaei, S. Dick, and W. Xu, "Toward non-intrusive load monitoring via multi-label classification," IEEE Transactions on Smart Grid, vol. 8, no. 1, pp. 26-40, Jan. 2017.

[7] H. Najmeddine, K. E. K. Drissi, C. Pasquier, C. Faure, K. Kerroum, A. Diop, T. Jouannet, and M. Michou, "State of art on load monitoring methods," in 2008 IEEE 2nd International Power and Energy Conference, Dec. 2008, pp. 1256-1258.

[8] S. R. Depuru, L. Wang, V. Devabhaktuni, and N. Gudi, "Smart meters for power grid challenges, issues, advantages and status," in IEEE/PES Power Systems Conference and Exposition, 2011.

[9] "The energy detective," http://www.theenergydetective.com/

[10] S. R. Depuru, L. Wang, V. Devabhaktuni, and N. Gudi, "System and method to monitor and manage performance of appliances," in U.S. Patent 8649987 B2, Feb. 2014.

[11] "Qualisteo wattseeker solution," http:/qualisteo.com/ws/ nialm-et-desagregation-courbe-de-charge/

[12] "Eyedro," http://eyedro.com/

[13] "Neurio,", http://neur.io

[14] "Sense," http://sense.com

[15] K. Gomez, R. Riggio, T. Rasheed, D. Miorandi, and F. Granelli, "Energino: a hardware and software solution for energy consumption monitoring," in $10^{\text {th }}$ Int.Symposium on Modeling and Optimization in Mobile, Ad Hoc and Wireless Networks (WiOpt), 2012, pp. 311-317.

[16] G. Aurilio, D. Gallo, C. Landi, M. Luiso, and G. Graditi, "A low cost smart meter network for a smart utility," in IEEE Int. Conf. On Instrumentation and Measurement Technology (I2MTC), 2014, pp. 380 385.

[17] M. C. Lorek, F. Chraim, K. S. J. Pister, and S. Lanzisera, "Cost-based stick-on electricity meters," IEEE Sensors Journal, vol. 14, no. 10, pp. 3482-3489, Feb. 2014.

[18] "LEM LV-25 P voltage transducer datasheet," https://www.lem.com/ sites/default/files/products_datasheets/lv_25-p.pdf

[19] "Arduino MKR zero technical specifications," https://store.arduino.cc/ arduino-mkrzero

[20] "SD memory cards: The features and specifications," https://www.macworld.com/article/3208766/storage/ sd-memory-cards-the-features-and-specifications-to-look-for.html

[21] S. Houidi, F. Auger, H. B. A. Sethom, L. Miègeville, D. Fourer, and X. Jiang, "Statistical assessment of abrupt change detectors for nonintrusive load monitoring," in 2018 IEEE International Conference on Industrial Technology (ICIT), Feb 2018, pp. 1314-1319.

[22] S. Houidi, F. Auger, H. B. A. Sethom, D. Fourer, and L. Miègeville, "Relevant feature selection for home appliances recognition," in Proc. of Electrimacs Conf., Jul 2017.

[23] "NILM data acquisition software," http://www.univ-nantes.fr/auger-f accessed: March 4, 2019. 